\title{
Le projet français P.I.V.E.R.T.
}

Jean-François ROUS

SAS P.I.V.E.R.T.,

11, Rue de Monceau,

CS 60003

75378 Paris

Cedex 08,

France

$<$ j.rous@prolea.com>

Article reçu le 12 juillet 2012

Accepté le 17 octobre 2012

\begin{abstract}
The P.I.V.E.R.T. project
P.I.V.E.R.T. is an ambitious project of development of the $3 \mathrm{rd}$ generation biorefinery by 2020. Conceived around a technological complex with strict environmental criteria (aiming at obtaining a label HQE), P.I.V.E.R.T. federates all the national and main roads lifeblood, of the sector (some international partnerships are also under development). The major objectives of the P.I.V.E.R.T. project are: a valorization of oilseeds, proteins and lignocellulosic plant in its entirety (approach "entire plants"); an operation in closed circuit on the level of water and energy exchanges, the platform providing for its own needs, in a logic of industrial ecology; collection and valorization of a significant part of the $\mathrm{CO}_{2}$ produced by the activities of the refinery. Two strategies will be developed jointly: on one hand the development of "platform" molecules entering the traditional circuits of chemistry, on the current markets with the renewable character, in addition, the development of new molecules having new properties, thus giving access to new markets. Thanks to the research and development realized by researchers dedicated to P.I.V.E.R.T., the industrial partners will have access to a capable structure to develop, and to test demonstrators preceding future industrial production units. Certain technological bricks could be tested in a large scale industrial platform dedicated to operational integration of innovations. The development of programming technologies of refineries will give to P.I.V.E.R.T. the means to develop new innovative processes and will give to the industrials the possibility of modeling their future production units.
\end{abstract}

Key words: P.I.V.E.R.T., project, 3rd generation biorefinery, valorization, indistrial platform, innovation

industriels et la limitation de leur impact sur l'environnement. Concrètement, la France s'est fixée des objectifs particulièrement ambitieux et le Grenelle de I'Environnement prévoit d'ici à 2020 (Pignault, 2009) 600000 emplois créés et 450 milliards d'euros cumulés de 2009 à 2020.

\section{Présentation de I'IEED (Institut d'excellence en énergie décarbonnée) P.I.V.E.R.T.}

La chimie du végétal : une chimie plus verte

La chimie du végétal est la production de produits chimiques à partir de ressources renouvelables agricoles et forestières et succède à une chimie fondée sur l'utilisation de ressources non renouvelables, le charbon (carbochimie) puis le pétrole (pétrochimie). C'est la nouvelle chimie économiquement durable. Alors que depuis dix ans l'emploi dans l'industrie chimique diminue car elle se délocalise vers les pays producteurs de pétrole ou de gaz, la chimie du végétal pourrait permettre de relocaliser la production à côté des ressources agricoles et forestières.

Pour se développer, l'industrie chimique doit pouvoir compter sur une base de ressources abondantes. Ainsi, la carbochimie, puis la pétrochimie se sont développées sur le charbon, puis le pétrole, car la disponibilité de ces matières premières était assurée. En effet, les besoins énergétiques et de transport modernes ont poussé l'exploi-

Pour citer cet article : Rous JF. Le projet français P.I.V.E.R.T. OCL $2012 ; 19(6)$ : 370-378. doi : 10.1684/ocl.2012.0432 
tation d'énormes quantités de charbon puis du pétrole, permettant à l'industrie chimique de se développer avec quelques pourcentages de ces quantités. La chimie du végétal s'appuie sur l'agriculture qui fournit des ressources renouvelables et des développements important liés à l'alimentation et aux besoins énergétiques. Les produits obtenus sont issus des huiles et de l'amidon ou des sucres.

La chimie du végétal n'est pas en compétition avec l'alimentation car elle utilise une faible part des terres arables. En 2006, 6000 millions de tonnes de ressources renouvelables ont été utilisés par l'homme comme suit : $62 \%$ pour l'alimentation, $33 \%$ pour le bois de construction, le papier et le bois de chauffage et $5 \%$, soit 300 millions de tonnes, pour la chimie et l'industrie (Kamm, 2006).

\section{Des atouts compétitifs pour la France}

Le principal atout de la France est de regrouper une position forte en agriculture et en chimie : la France est le deuxième acteur européen de la chimie avec un chiffre d'affaire de 68 milliards d'euros en 2009 (15\% des ventes européennes et cinquième acteur mondial). Cependant ce nombre décroît de $2 \%$ par an depuis 10 ans. L'ambition économique est de prendre le leadership du marché de la chimie du végétal, marché mondial de l'ordre de 300 milliards d'euros en 2020, avec une croissance estimée de $10 \%$ à $15 \%$ par an. Cependant, peu de choses sont structurées en France, dans la filière des oléagineux.

\section{Des enjeux technologiques identifies}

Le segment le plus prometteur pour la chimie du végétal est la chimie fine et de spécialités dont la valeur ajoutée permet de financer les innovations nécessaires (Carole et al., 2004). Les applications sont déjà nombreuses : solvants pour les encres et peintures, adhésifs et colles, huiles et graisses de lubrification, surfactants et tensio-actifs, applications des lipides dans les cosmétiques, etc. Les segments les plus volumineux et donc de moindre valeur ajoutée, les plastiques et la chimie de base, se développeront en second lieu (Nowicki et al., 2008) (tableaux 1 et 2).

\section{Les bioraffineries}

La bioraffinerie repose sur un ensemble de bio-industries intégrées. Elle utilise un éventail de technologies pour fabriquer des produits chimiques, des biocarburants, des biomatériaux, des aliments et ingrédients alimentaires, des fibres ainsi que de l'énergie sous forme de chaleur et d'électricité. Elle vise à maximiser la valeur ajoutée sur les trois piliers du développement durable: environnement, économie et société.

La France est un des pays européens les plus riches en biomasse (De Cherisey et al., 2007). À ce titre et pour réduire sa dépendance énergétique et ses émissions de gaz à effet de serre, elle s'est lancée dans un plan ambitieux de développement des biocarburants entraînant celui des bioraffineries de $1^{\text {re }}$ et $2^{\mathrm{e}}$ générations. Le nouvel enjeu industriel est de développer une $3^{\mathrm{e}}$ génération de bioraffineries qui combine différentes sources de matières premières pour produire de multiples produits chimiques, ainsi que des biocarburants ${ }^{1}$.

\section{La compétition internationale est ouverte}

Compte tenu de ces enjeux, la compétition internationale est engagée. Le département américain de l'énergie investit 700 millions de dollars pour les bioraffineries (703 millions de dollars de subvention en 2009 du DOE, département américain de l'énergie pour 19 projets de démonstration de bioraffineries). La Commission européenne a décidé de lancer un programme spécifique aux bioraffineries dans le cadre du $7^{\mathrm{e}}$ PCRD (Programme cadre de recherche et développement). Aux vues des investissements sur les différentes zones géographiques, I'ambition de I'IEED P.I.V.E.R.T. est de devenir le leader européen.

Ce leadership se développera par la maîtrise des procédés et l'efficacité économique. L'ambition de P.I.V.E.R.T. et des campus d'innovation technologique mondiaux est de développer les outils nécessaires pour améliorer la compétitivité économique et technique des bioraffineries. Ces performances viendront de I'optimisation des pro-

\footnotetext{
${ }^{1} \mathrm{cf}$. http://www.biorefinery-euroview.eu/ biorefinery/public/index.html
}

cédés de fermentation par batch ou en continu, de la maîtrise de la catalyse et de la biocatalyse, de la rationalisation des étapes de prétraitement, de la possibilité de transformer des matières premières végétales d'origine variées (résidus de l'agriculture, forêts, herbes, etc.) en un maximum de molécules.

Pour atteindre ces objectifs, I'IEED P.I.V.E.R.T. associe dans son fonctionnement et dans sa gouvernance, une $R \& D$ générique en amont, et une volonté forte $d^{\prime}$ 'industrialisation portée par les industriels, des chimistes, des équipementiers et des services d'ingénierie.

\section{Enjeux économiques et sociaux}

\section{Evaluation des marchés existants et de leur potentiel}

En Europe, le secteur de la chimie représente 449 milliards d'euros en 2009 (24\% des ventes mondiales) (Cefic, 2010). Sa croissance est estimée à $2 \%$ pour l'année 2011 et elle représente 1200000 emplois dont 180000 emplois directs en France (tableaux 3 et 4), correspondant à 1000000 d'emplois directs et indirects (le facteur des emplois indirects de l'industrie chimique est de 3 à 5 selon les données du Grenelle de l'Environnement, de 5,5 selon l'ACC). De par sa dépendance au pétrole, l'industrie chimique est menacée car très dépendante du prix et de la disponibilité du pétrole, ce qui explique son actuelle délocalisation vers les pays producteurs de pétrole ou de gaz. Ses coûts principaux sont ainsi répartis : $12 \%$ de coûts salariaux; $28 \%$ de coûts d'énergie ; $42 \%$ de coûts de matières premières en grande majorité d'origine pétrolière (Cefic Chemdata International, 2010). La chimie du végétal utilise quant à elle, des ressources agricoles et forestières. Elle constitue ainsi une alternative avec des matières premières renouvelables, contrôlées et locales.

La chimie du végétal pourrait permettre donc de pérenniser et de développer des emplois verts sur des marchés biosourcés en croissance, et surtout non délocalisables à terme. D'après différentes sources d'information, le marché de la chimie du végétal au niveau mondial est de l'ordre de 300 milliards d'euros à horizon 2020, avec une 


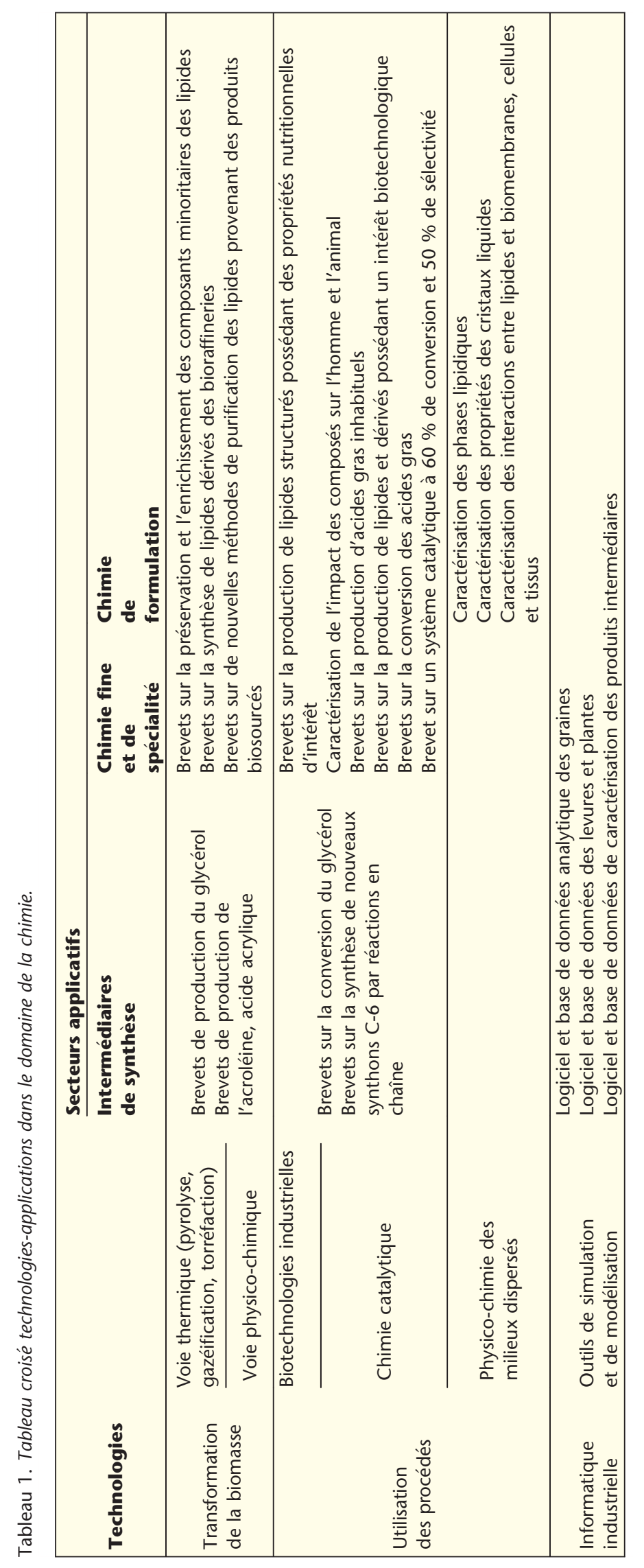

372 OCL VOL. 19 N 6 novembre-décembre 2012 
Tableau 2. Tableau croisé technologies-applications dans le domaine des équipements et de l'ingénierie industrielle.

\begin{tabular}{|c|c|c|c|}
\hline \multirow[t]{2}{*}{ Technologies } & & \multicolumn{2}{|l|}{ Secteurs applicatifs } \\
\hline & & $\begin{array}{l}\text { Equipements liés aux } \\
\text { développements des } \\
\text { technologies }\end{array}$ & $\begin{array}{l}\text { Services et ingénierie } \\
\text { industrielle }\end{array}$ \\
\hline \multirow{2}{*}{$\begin{array}{l}\text { Transformation } \\
\text { de la biomasse }\end{array}$} & $\begin{array}{l}\text { Voie thermique (pyrolyse, } \\
\text { gazéification, torréfaction) }\end{array}$ & $\begin{array}{l}\text { Brevets sur des procédés } \\
\text { thermochimiques optimisés }\end{array}$ & \multirow{5}{*}{$\begin{array}{l}\text { Développement des connaissances } \\
\text { pour les calculs de simulation } \\
\text { et de programmation linéaire de } \\
\text { la bioraffinerie de } 3^{\mathrm{e}} \text { génération }\end{array}$} \\
\hline & Voie physico-chimique & $\begin{array}{l}\text { Brevets sur des nouveaux } \\
\text { procédés de prétraitement }\end{array}$ & \\
\hline \multirow{3}{*}{$\begin{array}{l}\text { Utilisation des } \\
\text { procédés }\end{array}$} & Biotechnologies industrielles & $\begin{array}{l}\text { Développement d'équipements } \\
\text { spécifiques pour les biotechnologies } \\
\text { industrielles }\end{array}$ & \\
\hline & Chimie catalytique & $\begin{array}{l}\text { Brevet sur des systèmes catalytiques } \\
\text { fonctionnant sans solvant ou avec } \\
\text { des solvants éco-responsables } \\
\text { Brevets sur l'implantation du CO2 } \\
\text { comprimé pour la catalyse }\end{array}$ & \\
\hline & $\begin{array}{l}\text { Physico-chimie des milieux } \\
\text { dispersés }\end{array}$ & $\begin{array}{l}\text { Brevets sur des méthodes de purification } \\
\text { et/ou de production de lipides structurés }\end{array}$ & \\
\hline $\begin{array}{l}\text { Informatique } \\
\text { industrielle }\end{array}$ & $\begin{array}{l}\text { Outils de simulation } \\
\text { et de modélisation }\end{array}$ & $\begin{array}{l}\text { Logiciel de modélisation des } \\
\text { phénomènes } \\
\text { induits par les nouveaux } \\
\text { prétraitements } \\
\text { Logiciel de modélisation du pressage } \\
\text { sous conditions intensifiées } \\
\text { Logiciel de modélisation } \\
\text { d'ultrafiltration dynamique }\end{array}$ & $\begin{array}{l}\text { Logiciel de test pour évaluer } \\
\text { et adapter les performances } \\
\text { environnementales des } \\
\text { bioraffineries implantées } \\
\text { Evaluation et prévention des risques } \\
\text { industriels } \\
\text { Modélisation conceptuelle pour l'évaluation } \\
\text { environnementale de la bioraffinerie }\end{array}$ \\
\hline
\end{tabular}

croissance annuelle de $10 \%$ à $15 \%$ (tableau 5). Selon le Grenelle de I’Environnement et le département américain de l'Agriculture, la chimie du végétal représente $4 \%$ de la chimie dans le monde. Cette part passerait à $13 \%$ de la chimie en 2020 et constituerait un marché de 330 milliards d'euros. À l'échelle française et européenne, pour les industriels chimistes partenaires de I'Institut P.I.V.E.R.T., les matières premières renouvelables représentent en moyenne $8 \%$ de leurs achats. Ce taux devrait passer aux alentours de $15 \%$ d'ici 2020, et le chiffre d'affaires alors dégagé grâce à la chimie du végétal devrait représenter plus de 5 milliards d'euros. Il est estimé que P.I.V.E.R.T. sera contributeur d'une part significative de ce chiffre. La chimie du végétal ne représente en 2009 que 33000 emplois directs en France et atteindra 95000 emplois à I'horizon 2020 ce qui correspond à une croissance annuelle de
$10 \%$ et 62000 nouveaux emplois. Ces emplois représentent une opportunité pour la France en opposition aux pertes actuelles de l'industrie chimique française prévoyant une suppression de 30000 postes d'ici $2020(-17 \%$ d'ici à 2020) (CARMEE, 2009) (tableau 5).

En outre, il ne faut pas oublier que, parallèlement aux industries de l'agroalimentaire, de la chimie et de l'énergie, les ingénieurs européens ont développé

Tableau 3. Emplois et chiffre d'affaires de l'industrie chimique (France, Europe, Monde)

\begin{tabular}{|llll|}
\hline $\begin{array}{l}\text { Zone } \\
\text { géographique }\end{array}$ & $\begin{array}{l}\text { Chiffre d'affaires } \\
\text { de la chimie } \mathbf{2 0 0 9}\end{array}$ & $\begin{array}{l}\text { Chiffre d'affaires } \\
\text { de la chimie 2020 }\end{array}$ & $\begin{array}{l}\text { Emplois directs de FI'industrie } \\
\text { chimique 2009 }\end{array}$ \\
\hline France & $68 \mathrm{G}$ & $\begin{array}{l}85 \mathrm{G} € \\
(2 \% / \mathrm{an})\end{array}$ & $180000^{\mathrm{a}}$ \\
\hline Europe & $449 \mathrm{G} €$ & $\begin{array}{l}560 \mathrm{G} € \\
(2 \% / \mathrm{an})\end{array}$ & $\begin{array}{l}1200000 \\
-2 \% \mathrm{~d}^{\prime} \mathrm{emplois} / \mathrm{an} \text { depuis } 10 \text { ans }\end{array}$ \\
\hline États-Unis & & $\begin{array}{l}480 \mathrm{G} € \\
\left(3 \% / \mathrm{an}{ }^{\mathrm{b}}\right)\end{array}$ & $\begin{array}{l}800000 \mathrm{c}^{\mathrm{c}} \\
-2 \% \mathrm{~d} \text { emplois/an depuis } 10 \text { ans }\end{array}$ \\
\hline Monde & $347 \mathrm{G} €$ & $\begin{array}{l}2600 \mathrm{G} € \\
(3 \% / \mathrm{an})\end{array}$ & 5000000 \\
\hline
\end{tabular}

a Union des industries chimiques (UIC).

${ }^{b}$ USDA - Biobased products : market potential, 2008.

' Bureau of Labor Statistics-Employment, 2009, et American Chemistry Council (ACC). 
Tableau 4. Chiffre d'affaires de la chimie du végétal (France, Europe, Monde).

\begin{tabular}{|llll|}
\hline $\begin{array}{l}\text { Zone } \\
\text { géographique }\end{array}$ & $\begin{array}{l}\text { Part } 2009 \text { de la } \\
\text { chimie du végétal } \\
\text { (\% de la chimie) }\end{array}$ & $\begin{array}{l}\text { Part 2020 de la } \\
\text { chimie du végétal } \\
\text { (\% de la chimie) }\end{array}$ & $\begin{array}{l}\text { Croissance de la } \\
\text { chimie biosourcée }\end{array}$ \\
\hline France & $3 \mathrm{G} €(4 \%)$ & $14 \mathrm{G} €(15 \%)^{\mathrm{a}}$ & $15 \% / \mathrm{an}^{\mathrm{b}}$ \\
\hline Europe & $20 \mathrm{G} €(4 \%)^{\mathrm{c}}$ & $80 \mathrm{G} €(14 \%)$ & $13 \% / \mathrm{an}$ \\
\hline États-Unis & $14 \mathrm{G} €(4 \%)^{\mathrm{d}}$ & $75 \mathrm{G} €(15 \%)$ & $15 \% / \mathrm{an}$ \\
\hline Monde & $80 \mathrm{G} €(4 \%)$ & $330 \mathrm{G} €(14 \%)$ & $10-15 \% / \mathrm{an}$ \\
\hline
\end{tabular}

${ }^{a} G$ renelle de l'environnement et déclaration des industriels partenaires de P.I.V.E.R.T.

b ${ }^{\prime}$ 'objectif du Grenelle de l'Environnement est de passer de 5,3\% en 2005 à $15 \%$ en 2015 en utilisation de matières premières renouvelables.

'Six Lead Market Initiative, Agence exécutive pour la compétitivité et l'innovation (AECI).

${ }^{\mathrm{d}}$ Biotechnology industy organisation (BIO) - Biobased chemicals and products.

un marché d'équipements, de machines et de procédés industriels. Pour l'industrie chimique (qui représente $11 \%$ de ce marché européen, selon l'association européenne Orgalime, European Engineering Industries Association), les fournisseurs d'équipements et de machines représentent un marché européen de 20 milliards d'euros. 600 entreprises françaises y occupent une position forte avec 30000 emplois directs et un chiffre d'affaires de 5 milliards d'euros (GIFIC, 2009). Ce domaine réalise $45 \%$ de sa valeur à l'exportation, démontrant une expertise technique reconnue. En parallèle, en Europe, le marché des services en ingénierie industrielle (ingénierie pour les infrastructures, les installations industrielles et services d'ingénierie spécialisés) représente 70 milliards d'euros (EFCA, 2009). La France occupe une position avancée avec 215000 emplois directs et un chiffre d'affaire de 36 milliards d'euros (Syntec-Ingénierie, 2008). Ce domaine connaît une croissance soutenue de $3 \%$ par an, et les exportations représentent $30 \%$ du chiffre d'affaires.

Cependant, malgré cet attrait pour la chimie du végétal, l'Europe est en retard par rapport à des pays comme les ÉtatsUnis ou le Brésil. Les projets de développement de bioraffineries y sont encore trop rares, et les investissements restent insuffisants pour permettre à la recherche de s'y atteler. De plus, les utilisateurs finaux ne sont pas prêts à dépenser plus, bien que la demande augmente. Le développement des marchés dépendra des efforts réalisés pour améliorer la compétitivité de la chimie du végétal. Le regroupement des différents types de partenaires privés et publics s'avère donc nécessaire pour permettre aux technologies de se développer.

Une évaluation et une démonstration des avantages environnementaux et socioéconomiques des bioproduits doivent être mises en place. Les bioproduits ne pourront s'imposer sur le marché et y gagner des parts que s'ils apportent la preuve d'un véritable avantage environnemental, économique et social. L'analyse du cycle de vie (ACV) est une méthodologie qui s'impose aujourd'hui pour mesurer les impacts environnementaux des produits (figure 1). Le pôle Industries \& Agro-Ressources (IAR) et ses partenaires ont souhaité créer un consortium pour répondre collectivement aux demandes des acteurs de la filière en ACV dans le secteur des agroressources. La démarche IAR-ACV vise à concevoir, mettre au point puis diffuser un guide méthodologique ACV durabilité appliqué aux filières agroressources ${ }^{2}$.

En conclusion, la chimie du végétal est un secteur en croissance qui apporte des emplois locaux: pour l'industrie chimique qui innove et diminue sa dépendance au pétrole; pour l'industrie des équipements et des machines industrielles pour la chimie; etpour les services en ingénierie industrielle qui se développent avec l'industrie associée. L'objectif de P.I.V.E.R.T. est d'être l'institut d'excellence qui développe ces marchés d'application.

\section{Débouchés de I'IEED P.I.V.E.R.T.}

P.I.V.E.R.T. trouve des applications dans les différents secteurs de la chimie, de l'agroalimentaire et de l'ingénierie

\footnotetext{
${ }^{2}$ http://www.iar-pole.com/fr/index02.php? page $=$ sousrubrique $\&$ id $=5$ \&idsousrub $=144$
}

industrielle. L'industrie des huiles et de la lipochimie que représente P.I.V.E.R.T. trouve aujourd'hui ses applications dans la chimie de spécialités et de formulation.

\section{Chimie de base}

Parmi les principaux intermédiaires de synthèse, on compte en particulier : le glycérol, du niveau d'importance de l'éthanol (Werpy et Petersen, 2004) comme molécule-plateforme avec des dérivés comme l'épichlorhydrine, et potentiellement le PDO (propan-1,3diol) ou le 3 HPA (acide hydroxypropionique); I'acide acrylique (colle, textile, peinture, revêtement, résines) ; I'acroléine (pharmacie, plastiques, cosmétique).

Les solvants issus du végétal ou agrosolvants seront utilisés dans les domaines des encres et peintures, de la détergence et des produits phytosanitaires par les partenaires chimistes ainsi que ces intermédiaires de synthèse.

\section{Chimie fine et de spécialités}

Les produits de la chimie de spécialité (additifs, catalyseurs, plastifiants, surfactants) en lien avec P.I.V.E.R.T. sont les suivants : les corps gras (acides/amines/ alcools gras) qui trouvent leurs applications dans la pharmacie, la cosmétique, l'alimentation, mais également dans la détergence, les peintures et colles, ou les bioproduits; les dérivés des triglycérides; les produits agroalimentaires intermédiaires, arômes, ingrédients et additifs alimentaires. On peut citer comme exemple I'acide linoléique conjugué (CLA) pour le contrôle de la prise de poids.

Ce domaine concerne particulièrement les partenaires chimistes et agrochimistes. 
Tableau 5. Situation économique et perspectives pour I'IEED P.I.V.E.R.T.

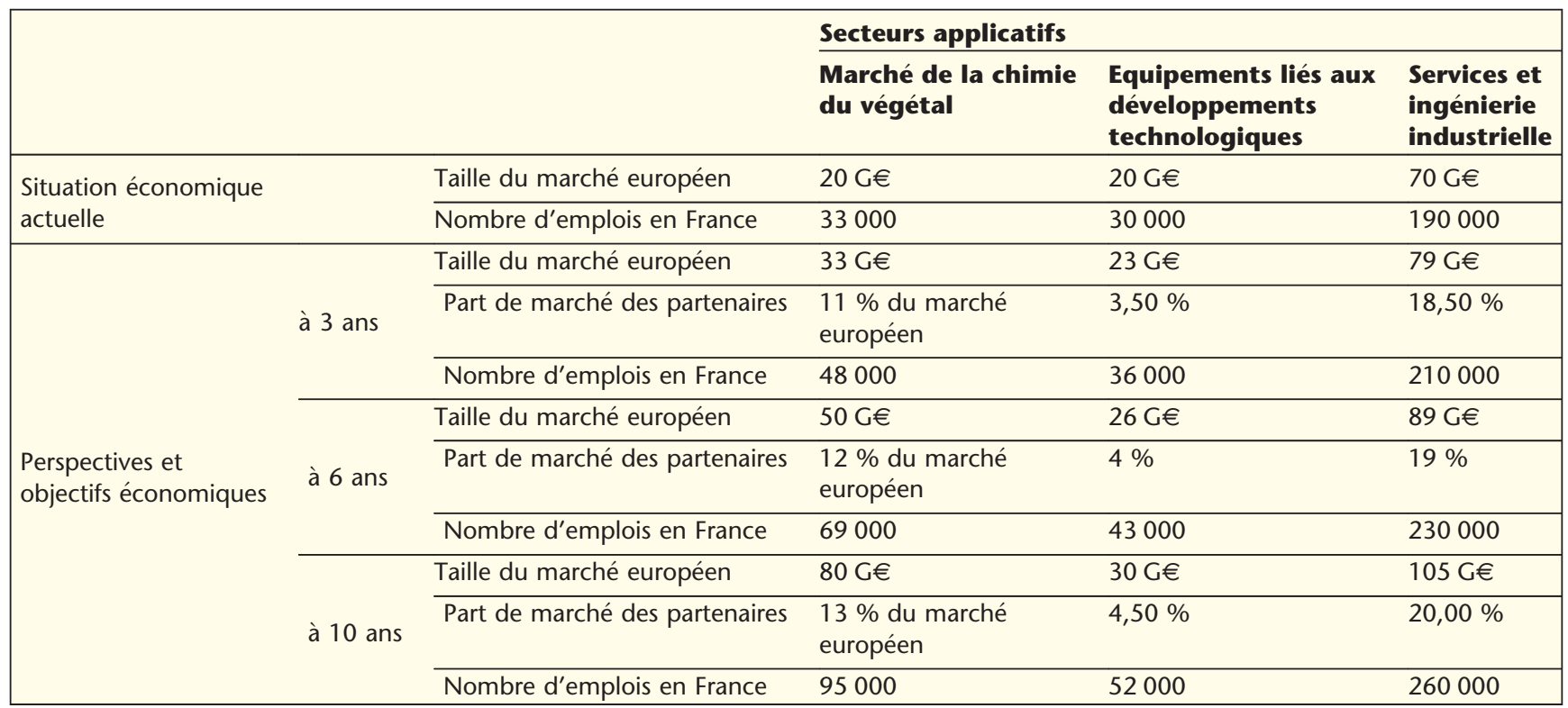

\section{Chimie de formulation}

La chimie de formulation sera ciblée par le développement de formules sur le site de P.I.V.E.R.T. Les débouchés seront les colles, les cosmétiques, les lubrifiants, les peintures, etc. Par exemple, des mélanges d'esters sont utilisés comme lubrifiants.

La chimie de spécialité et de formulation devrait représenter plus de des deux tiers de la chimie du végétal à horizon 2020 (tableau 6).

\section{Équipements et services}

Les équipements liés aux développements des technologies et les services et l'ingénierie industrielle seront étudiés et évolueront sur P.I.V.E.R.T. Des équipements innovants seront installés et renouvelés. En effet, les besoins de la chimie du végétal en nouveaux pro- cédés sont une source d'innovation pour les équipementiers. L'exportation des technologies liées à la bioraffinerie de troisième génération, voire même la construction de bioraffineries, constitue sur le long terme une voie d'application souhaitée pour les équipementiers partenaires de I'IEED. De la même façon, des acteurs de l'énergie y développent une expertise sur l'utilité et la valorisation énergétique de la biomasse.

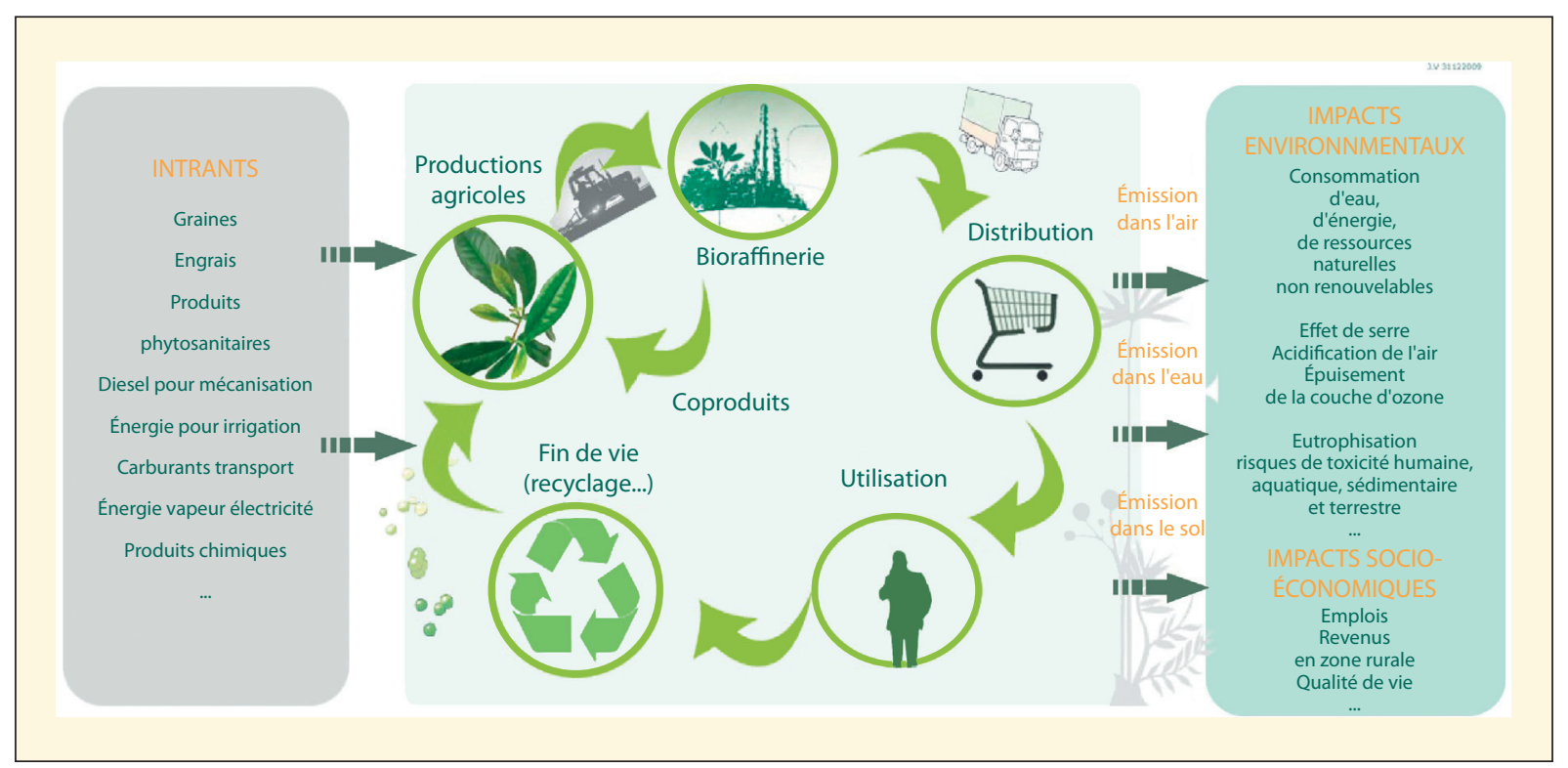

Figure 1. Démarche IAR-ACV. 
Tableau 6. Marchés de la chimie du végétal de 2009 à 2020 (Europe)

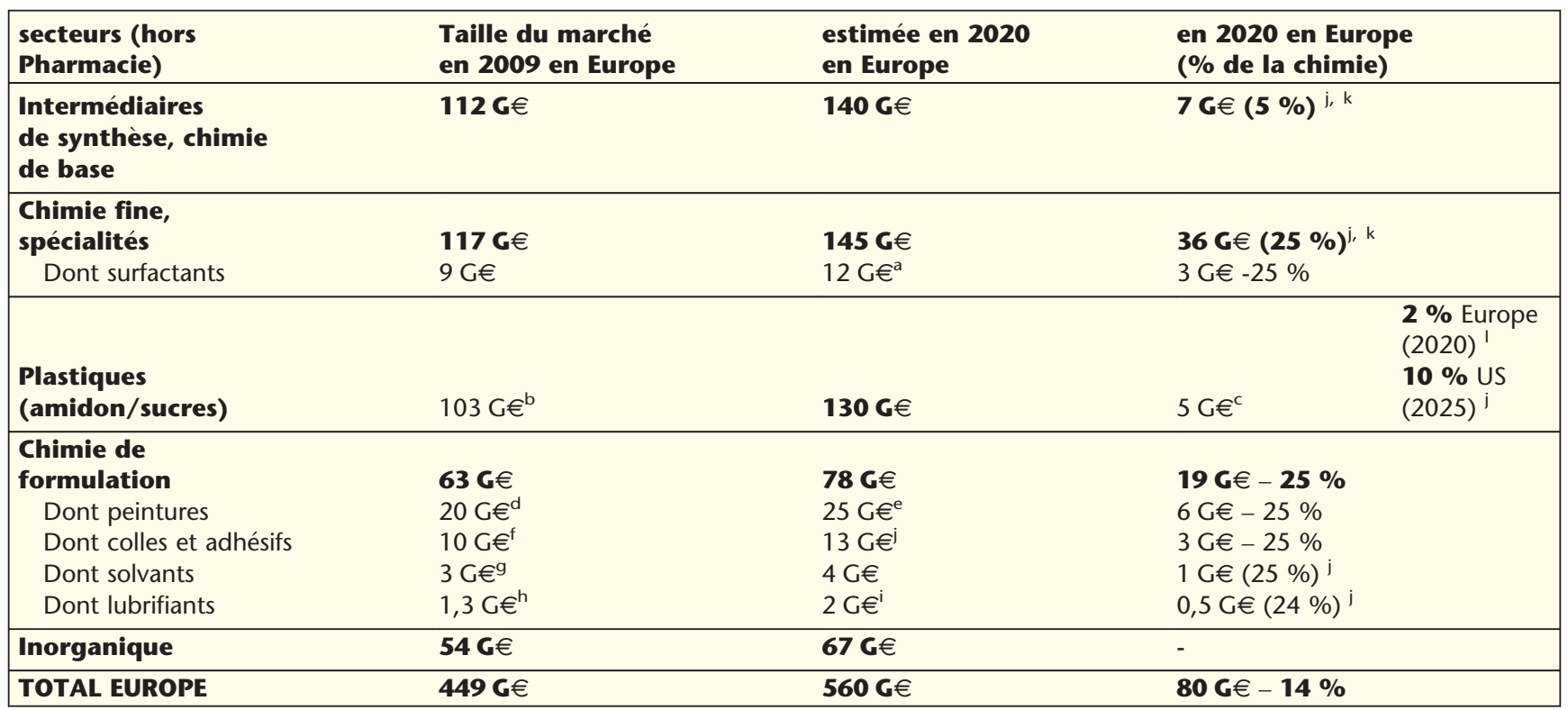

a World Surfactant market, Acmite Market Intelligence, 2008.

${ }^{b}$ La croissance des bioplastiques dans le monde est évaluée de 0,36 MT en 2007, 2,33 MT en 2013 et 3,45 MT en 2020.

c 1 million metric ton in 2020, PRO-BIP, 2009.

d 25-27 milliards de litres, soit 90 G \$ avec un croissance de 5 à $7 \%$ par an (Orr \& Boss ; Inc., 2007).

e $7 \%$ en Europe 2002-2007, 3 \% en Amérique du Nord, $13 \%$ en Asie - Prévisions 2008-2012 : 0,5\%/2 \%/9 \% (Fédération).

f Étude prospective sur le collage en France - DIGITIP - 2002.

g 2,5-3 G€, ESIG et CEFIC.

h www.carbohydrateeconomy.org/library/admin/uploadedfiles/Harvesting_Lubricants.htm

' Bio-based lubricants: a market opportunity study update, B. Bremmer \& L. Plonsker, 2008.

j USDA - Biobased products: market potential, 2008.

${ }^{k}$ Biotechnology industry organisation $(\mathrm{BIO})$ - Biobased chemicals and products.

' La croissance des bioplastiques dans le monde est évaluée de 0,36 MT en 2007, 2,33 MT en 2013 et 0,45 MT en 2020 pour une production mondiale de plastiques de 245 MT en 2007 (Plastics Europe, 2007).

\section{Objectifs et programmes de $R \& D$}

\section{Trois programmes synergiques}

P.I.V.E.R.T. comportera trois types de programmes, en synergie les uns avec les autres:

- un programme de recherche générique (GENESYS, figure 2) financé par les partenaires industriels et les Investissements d'Avenir dans un mode ouvert : les résultats seront accessibles à l'ensemble des partenaires ;

- des projets collaboratifs faisant intervenir les partenaires dans le cadre d'une relation contractuelle spécifique avec P.I.V.E.R.T. ;

- la genèse de la bioraffinerie de $3^{e}$ génération, le BIOGIS Center, véritable plateforme évolutive pour développer une stratégie de "métabolisme industriel » en s'appuyant sur les résultats des autres programmes, GENESYS en particulier. Cette conception de la bioraffinerie est en soi un programme de recherche et d'innovation.

Le programme GENESYS prendra son essor dès le début avec ses pleines capacités au cours des trois premières années. Les projets collaboratifs seront mis en place, et progressivement d'autres projets alimentés par les résultats de GENESYS suivront. Le volume de GENESYS connaîtra une décroissance progressive parallèle à la montée en puissance des projets collaboratifs des acteurs industriels. La genèse de la bioraffinerie P.I.V.E.R.T. sera alimentée par les résultats produits par le programme GENESYS et par l'essor des projets collaboratifs. Les équilibres entre les types de programmes permettront de maximiser les résultats pour l'essor de la filière et l'épanouissement de l'écosystème que constitue P.I.V.E.R.T., tout en respectant les intérêts de chacun des partenaires.

L'objectif principal de P.I.V.E.R.T., en particulier de GENESYS, est de jeter les bases d'une bioraffinerie pour les plantes oléagineuses et les lipides, par l'optimisation de toute la chaîne de production. Depuis la production des plantes avec l'agronomie, la récolte, la logistique jusqu'aux produits industriels biosourcés pour la chimie, les cosmétiques, la nutrition et la santé en passant par le fractionnement de la biomasse, la transformation et la formulation. La stratégie consiste à développer des approches innovantes et des procédés respectant les principes de la chimie verte. Les programmes de P.I.V.E.R.T. seront construits autour de trois fondements du développement durable: environnement, société et économie. Cette approche est complétée par l'évaluation des risques spécifiques de la démarche 


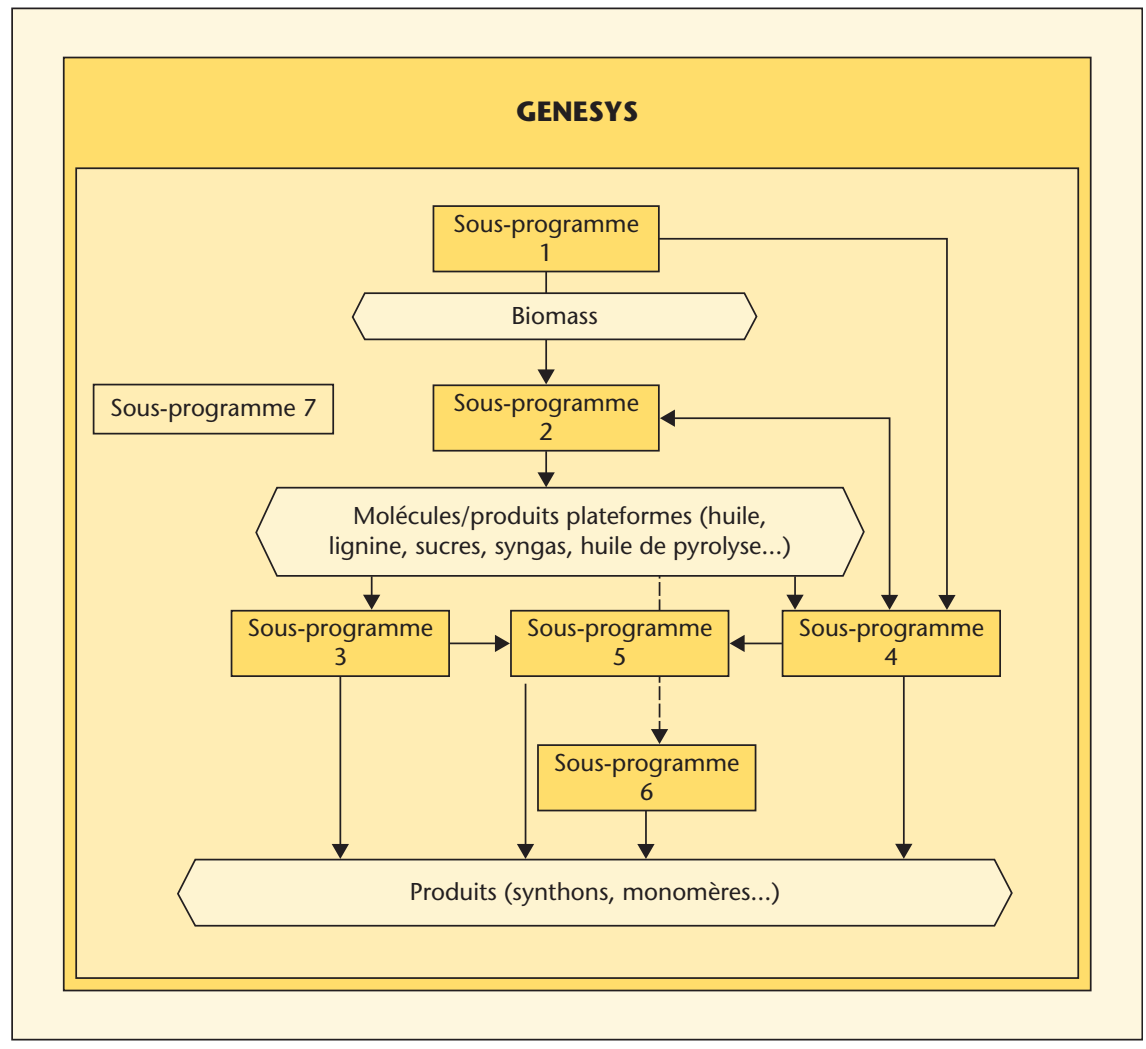

Figure 2. GENESYS, programme de recherche générique de P.I.V.E.R.T., financé par les partenaires industriels et les Investissements d'Avenir dans un mode ouvert.

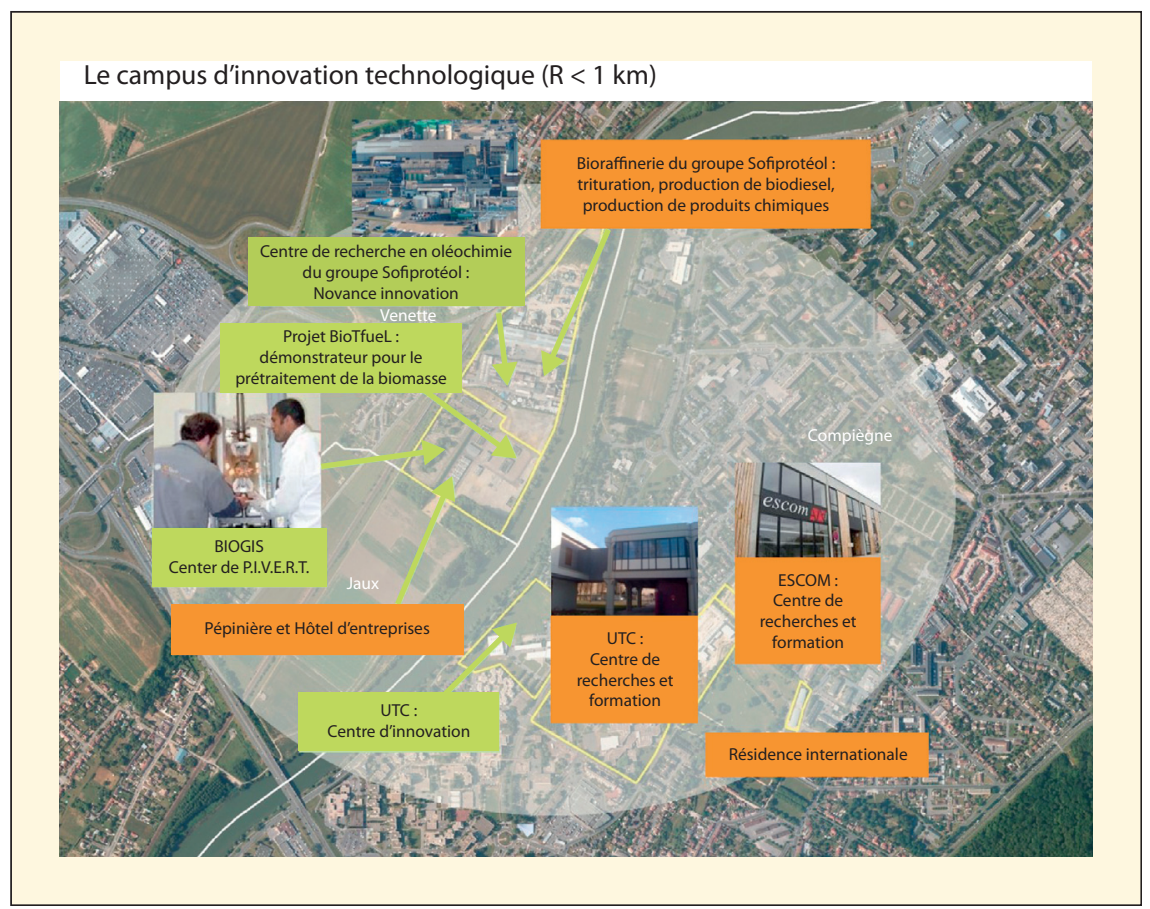

Figure 3. Implantation du BIOGIS Center, plateforme technologique de P.I.V.E.R.T., au sein du campus d'innovation technologique $(R<1 \mathrm{~km})$. proposée. Une analyse des cycles de vie sera réalisée en intégrant toutes les étapes depuis les productions de la biomasse jusqu'aux produits finaux et la bioraffinerie des oléagineux sera modélisée dans une logique de " métabolisme industriel ".

\section{7 secteurs scientifiques concernés}

Les secteurs scientifiques concernés par les programmes de P.I.V.E.R.T. seront au nombre de sept :

- I'adaptation, la mobilisation et la complémentation des agroressources oléagineuses ;

- les procédés physiques et thermochimiques de fractionnement de la biomasse ;

- la catalyse et la biocatalyse pour I'oléochimie, avec la mise en place de nouvelles réactions chimiques catalysées homogènes, hétérogènes ou enzymatiques;

- le métabolisme des lipides, de la plante aux microorganismes ;

- I'auto-assemblage des lipides pour la formulation et les créations de nanoobjets ;

- les nouveaux procédés pour obtenir des lipides d'intérêt pour la nutrition et la santé ;

- la prise en compte de l'écologie industrielle pour la réalisation de la bioraffinerie des oléagineux.

\section{Implantation du BIOGIS Center, plateforme technologique de P.I.V.E.R.T.}

Le site retenu pour l'implantation de la plateforme BIOGIS se situe sur le Parc Technologique des Rives de I'Oise, à côté du site industriel de Sofiprotéol (figure 3). Ce site a été choisi pour :

- sa proximité avec des centres de recherches publics et privés et d'un centre industriel ayant des activités en chimie du végétal ;

- les possibilités d'extension du site ;

- la disponibilité des sources d'énergie ;

- la possibilité d'avoir accès à une station de traitement des effluents liquides ;

- l'environnement du site sensibilisé aux contraintes industrielles ;

- les infrastructures d'accès au site déjà existantes ; 
- la présence du Parc Technologique des Rives de l'Oise dont les activités sont cohérentes et complémentaires pour le développement à moyen et long terme du BIOGIS Center.

Conflits d'intérêts : JFR est salarié de Sofiprotéol.

\section{RÉFÉRENCES}

Carole TM, Pellegrino J, Paster MD. Opportunities in the Industrial Biobased Products Industry. In : M. Finkelstein, J.D. McMillan, B. H. Davison, B. Evans (Eds.), Biotechnology for Fuels and Chemicals-The Twenty-Fifth Symposium. Applied Biochemistry and Biotechnology 2004; vol. 113-116 (12 Issues), Humana press Inc.

Cefic. Facts and Figures: The European chemical industry in a worldwide perspective: 2010. Cefic Chemdata International, 2010.
Contrat d'Etudes Prospectives de l'Industrie Chimique "Emploi Compétences", CARMEE, 2009.

De Cherisey H, Roy C, Pouet JC (Eds.). La valorisation de la biomasse. Guide d'information à l'attention des administrations et des établissements publics. Mars 2007. La valorisation de la biomasse, ADEME, 2007.

GIFIC, Groupement interprofessionnel pour les fournisseurs des industries chimique, FIM, Fédération des Industries Mécaniques, 2009.

EFCA, European Federation of Engineering Consultancy Associations, 2009.

Harlem Brundtland G (Eds.). Notre avenir à tous. Rapport de la Commission Mondiale sur I'Environnement et le Développement de I'ONU, présidée par Madame Gro Harlem Brundtland, avril 1987.

Kamm B, R. Gruber P, Kamm M. Industrial chemicals from biomass, In: Thoen et Busch (eds.), Biorefineries-Industrial Processes and Products: Status Quo and Future Directions. WILEY-VCH Verlag GmbH \& Co. KgaA, 2006.
Nowicki P, Banse M, Bolck C, Bos H, Scott E. Biobased economy. State-of-the-art assessment. LEI, The Hague, 2008.

Pignault G. Grenelle de l'environnement plan de mobilisation nationale sur les métiers liés à la croissance verte. Décembre 2009.

Stratégie nationale de développement durable. Stratégie nationale de développement durable 2010-2013 : vers une économie verte et équitable, 27 juillet 2010. http:// www.developpement-durable.gouv.fr/-Strategienationale-de,3900-.html.

Syntec-Ingénierie. Les derniers chiffres-clés de l'ingénierie. Publié le 10 décembre 2008. http://www.syntec-ingenierie.fr/actualites/ 2008/12/10/les-derniers-chiffres-cles-delingenierie-2008/.

Werpy T, Petersen G (Eds). Top Value Added Chemicals From Biomass. Volume I: Results of Screening for Potential Candidates from Sugars and Synthesis Gas. Department of Energy (DOE), USA, 2004. 